\title{
CONSTANT SUM PARTITION OF SETS OF INTEGERS AND DISTANCE MAGIC GRAPHS
}

\author{
Sylwia Cichacz AND AgnieszKa GőRlich \\ AGH University of Science and Technology \\ Faculty of Applied Mathematics \\ e-mail: cichacz@agh.edu.pl \\ forys@agh.edu.pl
}

\begin{abstract}
Let $A=\{1,2, \ldots, t m+t n\}$. We shall say that $A$ has the $(m, n, t)$-balanced constant-sum-partition property $((m, n, t)$-BCSP-property $)$ if there exists a partition of $A$ into $2 t$ pairwise disjoint subsets $A^{1}, A^{2}, \ldots, A^{t}, B^{1}, B^{2}, \ldots, B^{t}$ such that $\left|A^{i}\right|=m$ and $\left|B^{i}\right|=n$, and $\sum_{a \in A^{i}} a=\sum_{b \in B^{j}} b$ for $1 \leq i \leq t$ and $1 \leq j \leq t$. In this paper we give sufficient and necessary conditions for a set $A$ to have the $(m, n, t)$-BCSP-property in the case when $m$ and $n$ are both even. We use this result to show some families of distance magic graphs.
\end{abstract}

Keywords: constant sum partition, distance magic labeling, product of graphs.

2010 Mathematics Subject Classification: 05C78. 


\section{REFERENCES}

[1] Y. Alavi, A.J. Boals, G. Chartrand, P. Erdős and O.R. Oellerman, The ascending subgraph decomposition problem, Congr. Numer. 58 (1987) 7-14.

[2] K. Ando, S. Gervacio and M. Kano, Disjoint subsets of integers having a constant sum, Discrete Math. 82 (1990) 7-11. doi:10.1016/0012-365X(90)90040-O

[3] M. Anholcer and S. Cichacz, Note on distance magic products $G \circ C_{4}$, Graphs Combin. 31 (2015) 1117-1124. doi:10.1007/s00373-014-1453-x

[4] M. Anholcer, S. Cichacz, I. Peterin and A. Tepeh, Distance magic labeling and two products of graphs, Graphs Combin. 31 (2015) 1125-1136. doi:10.1007/s00373-014-1455-8

[5] S. Arumugam, D. Froncek and N. Kamatchi, Distance magic graphs - A survey, J. Indones. Math. Soc., Special Edition (2011) 11-26.

[6] S. Beena, On $\Sigma$ and $\Sigma^{\prime}$ labelled graphs, Discrete Math. 309 (2009) 1783-1787. doi:10.1016/j.disc.2008.02.038

[7] F.L. Chen, H.L. Fu, Y. Wang and J. Zhou, Partition of a set of integers into subsets with prescribed sums, Taiwanese J. Math. 9 (2005) 629-638.

[8] S. Cichacz, D. Froncek, E. Krop and C. Raridan, Distance magic Cartesian products of graphs, Discuss. Math. Graph Theory 36 (2016) 299-308. doi:10.7151/dmgt.1852

[9] H. Enomoto and M. Kano, Disjoint odd integer subsets having a constant even sum, Discrete Math. 137 (1995) 189-193. doi:10.1016/0012-365X(93)E0128-Q

[10] R.J. Faudree, A. Gyárfás and R.H. Schelp, Graphs which have an ascending subgraph decomposition, Congr. Numer. 59 (1987) 49-54.

[11] H.L. Fu and W.H. Hu, A note on ascending subgraph decompositions of complete multipartite graphs, Discrete Math. 226 (2001) 397-402. doi:10.1016/S0012-365X(00)00171-0

[12] H.L. Fu and W.H. Hu, A special partition of the set $I_{n}$, Bull. Inst. Combin. Appl. 6 (1992) 57-61.

[13] H.L. Fu and W.H. Hu, Ascending subgraph decompositions of regular graphs, Discrete Math. 253 (2002) 11-18. doi:10.1016/S0012-365X(01)00445-9

[14] H.L. Fu and W.H. Hu, Disjoint odd integer subsets having a constant odd sum, Discrete Math. 128 (1994) 143-150. doi:10.1016/0012-365X(94)90108-2

[15] J.A. Gallian, A dynamic survey of graph labeling, Electron. J. Combin. (2016) \#DS6. 
[16] P. Gregor and P. Kovář, Distance magic labelings of hypercubes, Electron. Notes Discrete Math. 40 (2013) 145-149. doi:10.1016/j.endm.2013.05.027

[17] T.R. Hagedorn, Magic rectangles revisited, Discrete Math. 207 (1999) 65-72. doi:10.1016/S0012-365X(99)00041-2

[18] F. Harary, Graph Theory (Addison-Wesley, Reading, MA, 1994).

[19] T. Harmuth, Über magische Quadrate und ähnliche Zahlenfiguren, Arch. Math. Phys. 66 (1881) 286-313.

[20] T. Harmuth, Über magische Rechtecke mit ungeraden Seitenzahlen, Arch. Math. Phys. 66 (1881) 413-447.

[21] R. Hammack, W. Imrich and S. Klavžar, Handbook of Product Graphs, Second Edition (CRC Press, Boca Raton, FL, 2011).

[22] D. Kotlar, Distance magic labeling in complete 4-partite graphs, Graphs Combin. 32 (2016) 1027-1038. doi:10.1007/s00373-015-1627-1

[23] A. Lladó and J. Moragas, On the sumset partition problem, Electron. Notes Discrete Math. 34 (2009) 15-19. doi:10.1016/j.endm.2009.07.003

[24] A. Lladó and J. Moragas, On the modular sumset partition problem, European J. Combin. 33 (2012) 427-434. doi:10.1016/j.ejc.2011.09.001

[25] K. Ma, H. Zhou and J. Zhou, On the ascending star subgraph decomposition of star forests, Combinatorica 14 (1994) 307-320. doi:10.1007/BF01212979

[26] M. Miller, C. Rodger and R. Simanjuntak, Distance magic labelings of graphs, Australas. J. Combin. 28 (2003) 305-315.

[27] A. O'Neal and P.J. Slater, Uniqueness of vertex magic constants, SIAM J. Discrete Math. 27 (2013) 708-716. doi:10.1137/110834421

[28] S.B. Rao, T. Singh and V. Prameswaran, Some sigma labelled graphs I, in: Graphs, Combinatorics, Algorithms and Applications, S. Arumugam, B.D. Acharya and S.B. Rao, (Eds.), (Narosa Publishing House, New Delhi, 2004) 125-133.

[29] V. Vilfred, $\Sigma$-Labelled Graphs and Circulant Graphs (Ph.D. Thesis, University of Kerala, Trivandrum, India, 1994).

Received 25 February 2016

Revised 7 October 2016

Accepted 8 October 2016 